Review article

\title{
Growing evidence supporting the use of mesenchymal stem cell therapies in multiple sclerosis: A systematic review
}

\author{
Ana Gabriela Oliveira $^{\mathrm{a}, 1}$, Margarida Gonçalves ${ }^{\mathrm{a}, 1}$, Helena Ferreira ${ }^{\mathrm{b}, \mathrm{c}, *}$, Nuno M. Neves ${ }^{\mathrm{b}, \mathrm{c}, \mathrm{d}, *}$ \\ ${ }^{a}$ Medicine School, University of Minho, Campus de Gualtar, Braga 4710-057, Portugal \\ b $3 B$ 's Research Group, I3B's - Research Institute on Biomaterials, Biodegradables and Biomimetics, University of Minho, Headquarters of the European Institute of \\ Excellence on Tissue Engineering and Regenerative Medicine, Avepark, Parque de Ciência e Tecnologia, Zona Industrial da Gandra, Barco, Guimarães 4805-017, Portugal \\ ${ }^{\mathrm{c}}$ ICVS/3B's - PT Government Associate Laboratory, Braga, Guimarães, Portugal \\ ${ }^{\mathrm{d}}$ The Discoveries Centre for Regenerative and Precision Medicine, Headquarters at University of Minho, Avepark, Barco, Guimarães 4805-017, Portugal
}

\section{A R T I C L E I N F O}

\section{Keywords:}

Mesenchymal stem cells

Multiple sclerosis

Adverse events

Therapeutic efficacy

Expanded disability status scale

\begin{abstract}
A B S T R A C T
Background: Multiple sclerosis (MS) typically arises in early/middle adulthood and is characterized by a progressive disability of the central nervous system (CNS). Currently approved therapies do not promote tissue repair or stop disease progression. Emerging data demonstrate that stem cells present a great potential in regenerative medicine and, consequently, have also been widely investigated as a potential treatment for MS. Therefore, the aim of this study was to conduct a systematic review to inquire into the safety, tolerability, and efficacy of mesenchymal stem cells (MSCs) therapies in MS.

Methods: Three electronic databases (Web of Science, PubMed, and Cochrane) were searched from April until June 2019. Clinical trials or case reports with information related to the effects of MSC therapies in MS patients were considered for this review.

Results: 10 manuscripts were selected, namely 7 uncontrolled clinical trials, 2 randomized controlled clinical trials, and 1 case report. The overall quality of the studies was considered good. Besides minor adverse events (AEs), it was reported one case of encephalopathy with seizures and two cases of iatrogenic meningitis, which were not related to the treatment, but with the administration route. The analyses of the expanded disability status scale (EDSS) in the uncontrolled clinical trials demonstrated that 48 patients improved, 39 maintained and 16 worsened their clinical condition. Regarding the randomized studies, one did not show statistically significant variations in the mean EDSS score and in the other the mean EDSS score was statistically significantly lower for the experimental group. The case report also showed an improvement in the EDSS score.

Conclusions: MSCs transplantation proved to be a safe and tolerable therapy. Their potential therapeutic benefits were also validated. However, larger placebo controlled blinded clinical trials will be required to establish the long term safety and efficacy profile of these therapies for MS. Their translation into the clinical practice can provide a new hope for the patients of this highly debilitating disease.
\end{abstract}

\section{Introduction}

Multiple Sclerosis (MS) affects ca.2.5 million people worldwide and is the main cause of chronic neurologic disability in young to middle adulthood (onset generally between the ages of 20 and 40 years) (Iwanowski and Losy, 2015). This disease is characterized by a progressive disability, due to gradual demyelination and variable axonal, neuronal and astroglial injury in the brain and/or spinal cord (Keegan and Noseworthy, 2002). MS presents mainly three different clinical courses (Iwanowski and Losy, 2015; Thompson et al., 2018).
Relapsing-remitting (RR)MS is the most frequent course (85-90\% of the MS patients) and is characterized by exacerbations followed by full or partial remissions (Iwanowski and Losy, 2015). In about 50-60\% of these patients, the disease evolves to secondary progressive (SP)MS, after many years, occurring a gradual worsening of the symptoms without remission (Giovannoni et al., 2016; Scalfari et al., 2014). Primary progressive $(\mathrm{PP}) \mathrm{MS}$ affects $\approx 15 \%$ of the patients and is characterized by a progressive dysfunction, having or not exacerbation episodes (Ontaneda et al., 2017). Unlike PPMS, RRMS and SPMS affects women more frequently (2.3-3.5:1) and more severely than men

\footnotetext{
* Corresponding authors.

E-mail addresses: helenaferreira@i3bs.uminho.pt (H. Ferreira), nuno@i3bs.uminho.pt (N. M. Neves).

${ }^{1}$ These authors contributed equally
} 


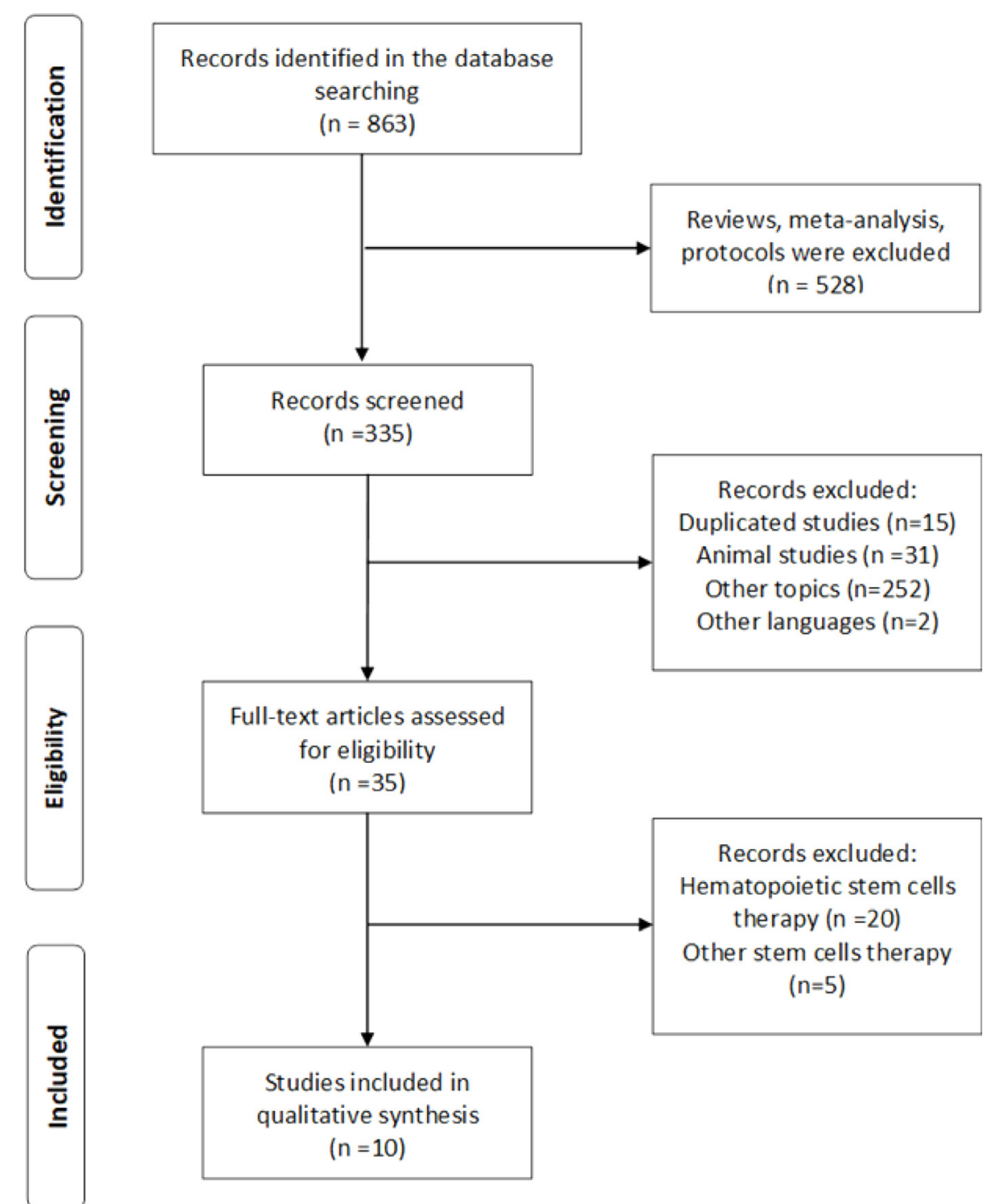

Fig. 1. Flow chart of the included and excluded studies.

(Ontaneda et al., 2017; Ramien et al., 2016; Harbo et al., 2013).

Currently approved therapies for MS (e.g. glatiramer acetate, natalizumab, mitoxantrone, cladribine, and ocrelizumab) are effective to prevent the accumulation of focal inflammatory damage and to reduce the number of relapses, but do not promote tissue repair and are not effective in stopping disease progression (Cohen et al., 2018; Li et al., 2014; Comi et al., 2017). Indeed, although important advances in MS treatment were achieved in the last decade, there is currently no cure for this highly debilitating condition. Additionally, these medications can present severe adverse effects, such as enhanced patient susceptibility to infections and cancer. Moreover, the therapeutic options for the more severe forms of the disease (PPMS and SPMS) are still very limited. However, the recognition of spontaneous remyelination in MS increased the success probability of the treatment strategies aimed at enhancing endogenous repair (Chandran and Compston, 2005; Prineas and Connell, 1979). Emerging evidence demonstrated that stem cells have great potential in regenerative medicine. Indeed, they present the potential to cure severe chronic conditions (Juric et al., 2016). Consequently, they may provide an effective and alternative therapy to the currently approved MS treatments (McLauchlan and Robertson, 2018). Stem cell therapies can be used to replace damaged or lost neuronal tissue, to replace the malfunctioning immune system, and to provide immunosuppressive, reparative and neuroprotective mechanisms (Shroff, 2018). The stem cells in general present ability to ameliorate the symptoms and pathological features of MS, but their selection should take into consideration different aspects, such as an easy collection, immunogenicity, the possibility of oncogenic transformation, among others. There are different types of human stem cells, such as neural stem cells (NSCs), embryonic stem cells (ESCs), induced pluripotent stem cells (iPSCs), hematopoietic stem cells (HSCs) and mesenchymal stem cells (MSCs). NSCs are present mainly in the subgranular zone of the dentate gyrus within the hippocampus and the subventricular zone (SVZ) of the lateral ventricles (Cuascut and Hutton, 2019). Despite promising therapeutic outcomes, one major limitation is the limited number of endogenous NSCs that can be obtained (Shroff, 2018; Cuascut and Hutton, 2019). Therefore, other types of stem cells, such as MSCs, iPSCs, and ESCs have been used to obtain NSCs (Shroff, 2018). The use of ESCs is controversial since they are pluripotent stem cells derived from sacrificed embryos (Shroff, 2018). Despite their ability to differentiate into any cell type of the body, the transplantation of ESCs in an experimental autoimmune encephalomyelitis (EAE) mice model demonstrated that the therapeutic effect was not related to graft or host remyelination, but due to an immunosuppressive neuroprotective mechanism (Aharonowiz et al., 2008). A case report (Shroff, 2015) demonstrated a significant improvement of the symptoms in a MS patient without related adverse 
Table 1

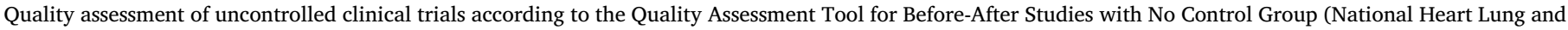
Blood Institute; see Appendix S1): good: $\square$; fair: $\square$; poor: $\square$.

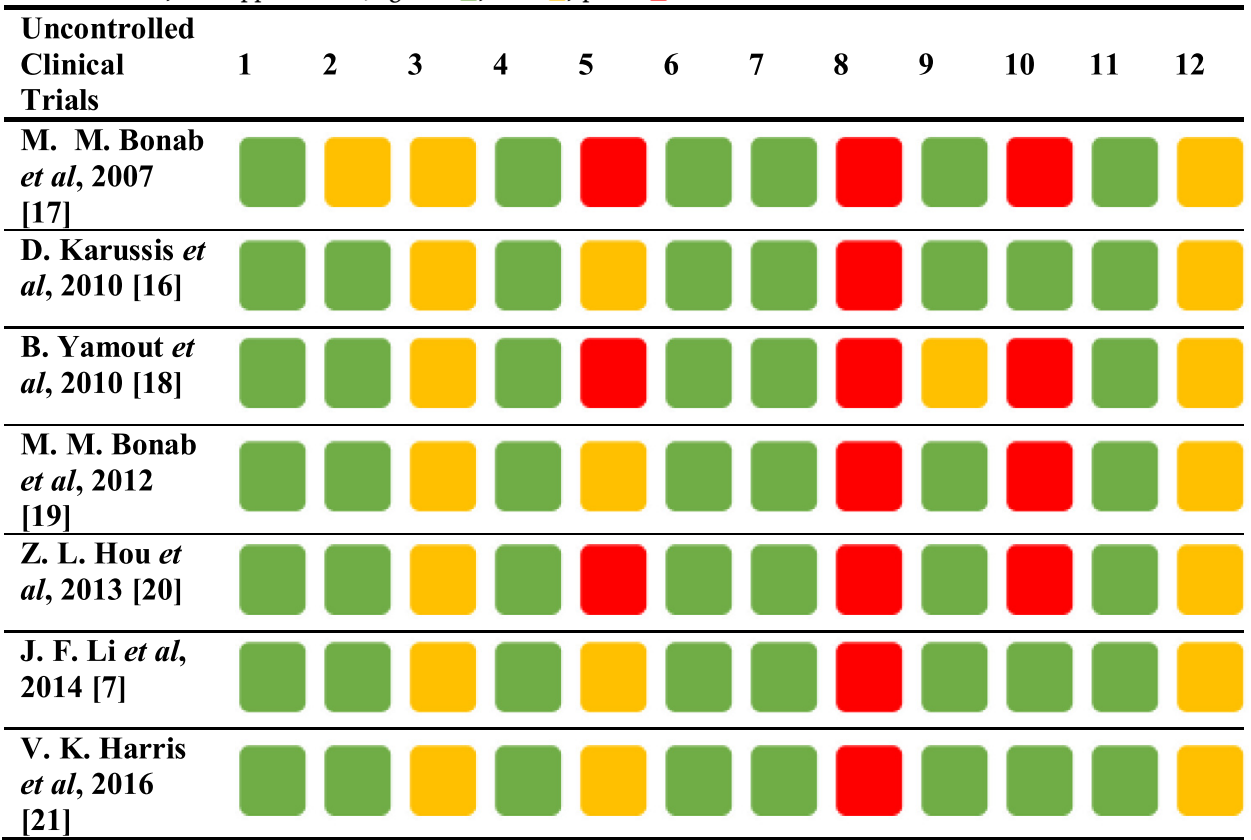

Table 2

Quality assessment of randomized clinical trials using the CASP checklist (see Appendix S1): good- [; fair- 0 .

\begin{tabular}{l|l|lllllllllll}
\hline $\begin{array}{l}\text { Randomized } \\
\text { Clinical } \\
\text { Trials }\end{array}$ & 1 & 2 & 3 & 4 & 5 & 6 & 7 & 8 & 9 & 10 & 11 \\
\hline $\begin{array}{l}\text { J. F. Li } \text { et } \text { al, } \\
2014[10]\end{array}$ &
\end{tabular}

Table 3

Quality assessment of Case Report using the checklist of BMJ Evidence-Based Medicine (see Appendix S1): good--

\begin{tabular}{llllllllll}
\hline Case Report & 1 & 2 & 3 & 4 & 5 & 6 & 7 & 8 \\
\hline Z. L. Hou et al, 2013 [37] & & & & & & & & & \\
\hline
\end{tabular}

effects. However, large scale studies are needed to support their efficacy and safety. In MS, HSCs transplant intends to replace the malfunctioning immune system after its ablation, and, consequently, the associated efficacy can be related to either aggressive immunosuppression or immune system alteration (McLauchlan and Robertson, 2018). Young patients with an inflammatory-active RRMS are those who can benefit more from this therapy. Indeed, $70-80 \%$ of RRMS patients after a HSCs transplant experienced a complete suppression of disease activity for 4-5 years (Muraro et al., 2017). iPSCs are generated from somatic cells and have similar potency to that of ESCs. Mouse iPSC-derived NSCs/neural precursor cells (NPCs) improved the symptoms of an EAE animal model due mainly to a neuroprotective effect than by remyelination (Laterza et al., 2013). However, the potential of iPSCs-derived oligodendrocyte progenitor cells in demyelinated axons demonstrated benefits in a non-human primate model (Thiruvalluvan et al., 2016). Despite their potential, some caution in their clinical use should be taken, due to the potential of immune rejection, genetic and epigenetic abnormalities, and tumour development (Okano et al., 2013). Adult-derived MSCs are the cell type that has been tested most consistently in neurological diseases, given their safety profile (Lukomska et al., 2019; Colpo et al., 2015; A Uccelli et al., 2011). MSCs are a heterogeneous subset of stromal progenitors of mesodermal cells with several advantages over the other stem cells, such as easy collection, high availability, easy culture methods, low immunogenicity allowing allotransplant, immunomodulatory ability, no oncogenic transformation, and minor ethical concerns. They can be isolated from various tissues, but bone marrow, and adipose tissues are the most widely used sources of MSCs (Zheng et al., 2017; Jorgensen and Noel, 2011). The MSCs administration in established animal models of MS demonstrated their potential to significantly improve the course of the pathology (Jiang et al., 2017; Bai et al., 2009; Yan et al., 2018; Kassis et al., 2008). Remarkable improvements of the MS clinical course after infusion of stem cells were obtained in clinical trials (Keirstead, 2005; Ardeshiry Lajimi et al., 2013). Although 

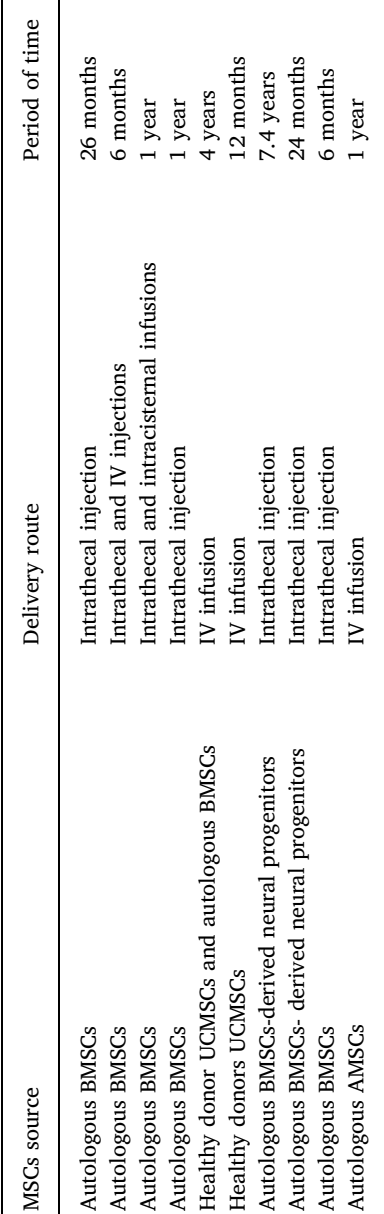

总

䒕

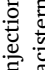

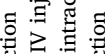

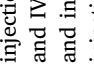

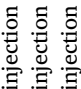

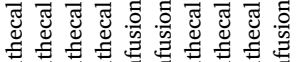

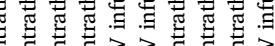

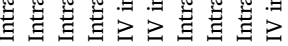

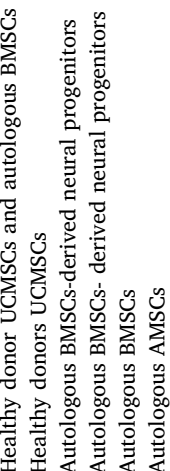

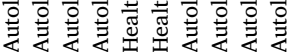
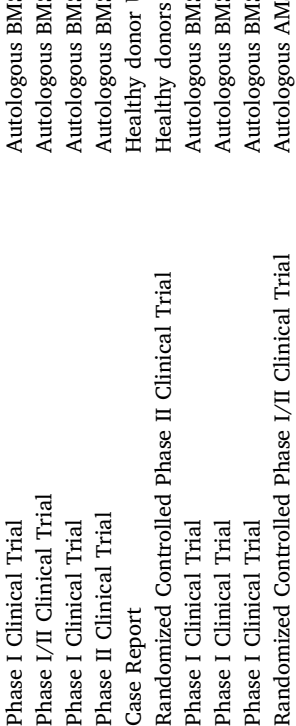

encouraging, some clinical trials indicate that the systemic injection of MSCs has major limitations, such as poor cell survival and low distribution into the CNS (A Uccelli et al., 2011). Consequently, other routes have been explored, such as intrathecal injections.

Due to the increasing importance of the use of stem cells in the treatment of MS, this systematic review investigates the safety, tolerability, and efficacy of MSC therapies in MS based in the scientific evidence available in the literature.

\section{Materials and methods}

This systematic review was conducted following the Preferred Reporting Items for Systematic Reviews and Meta-Analyses (PRISMA) guidelines.

\subsection{Search strategy}

Three electronic databases (Web of Science, PubMed and Cochrane) were used systematically from April to June 2019. The following keywords, Boolean operators, and combinations were used: "(Human "stem cell" AND Multiple Sclerosis)" NOT "Embryonic" NOT "Experimental autoimmune encephalomyelitis".

\subsection{Eligibility criteria}

Experimental studies with information related to the effects of stem cell therapies in MS patients were considered eligible. The clinical outcomes related to efficacy measures (e.g. expanded disability status scale -EDSS, relapse rate, whole-brain atrophy, and the number of gadolinium-enhancing lesions or T2-weighted lesions in the brain and spinal cord) and/or the adverse events (AEs) should be described. All trial studies and case reports were considered in the evaluation. The following records were excluded: a) reviews; b) meta-analysis; c) protocols; d) animal studies; e) other topics (studies that not include the use of stem cell therapies in the treatment of MS patients) and f) studies not written in English. After analyzing the full text of the eligible articles, studies about peripheral blood stem cell transplantation and records that do not specify the stem cells type and/or origin were excluded. Only manuscripts focusing on MSC therapies were included.

After analyzing the full text of the eligible articles, we included only manuscripts focusing on MSC therapies.

\subsection{Study selection}

Two reviewers independently analyzed the records initially screened according to the inclusion and exclusion criteria. After the agreement, full-text copies of every potentially relevant study were obtained. This methodology allowed the simultaneous analysis of the eligible manuscripts by the two authors. The manuscripts that fulfilled the inclusion criteria were selected for this systematic review.

\subsection{Data extraction}

Information about the author names, publication date, country, study type, time period of the study, characteristics of the participants, inclusion and exclusion criteria and outcomes concerning AEs and EDSS were independently collected by the two researchers in the original reports. The assessment of the improvement or worsening of MS patients is based on a set of clinical parameters (Section 2.2), but the EDSS score was the unique outcome parameter reported in all the selected papers, being consequently selected to evaluate the efficacy outcomes. Data treatment consisted in the calculation of means and percentages.

\subsection{Quality appraisal}

The quality assessment of the non-controlled studies was established 
Table 5

Characteristics of the participants of the selected studies.

\begin{tabular}{|c|c|c|c|c|c|c|}
\hline Authors, year & Patients number & Female number & Male number & Age & EDSS & Clinical Course \\
\hline Mohyeddin Bonab et al. (2007) & 10 & 7 & 3 & 22-40 Mean: 33 & 3.5 to 6.0 & 2 PPMS; 8 SPMS. \\
\hline Karussis et al. (2010) & 15 & 8 & 7 & Mean: 35.3 & 4.0 to 8.0 & ----- \\
\hline Yamout et al. (2010) & 7 & 4 & 3 & 34-56 Mean: 42.8 & 4.5 to 7.5 & 7 SPMS. \\
\hline Bonab et al. (2012) & 25 & 19 & 6 & 23-50 Mean: 34.7 & 3.5 to 7.0 & 2 PPMS; 23 SPMS. \\
\hline Hou et al. (2013) & 1 & 0 & 1 & 25 & ----- & 1 RRMS \\
\hline Li et al. (2014) & 23 & 16 & 7 & $\begin{array}{l}\text { Experimental group: } 41.7 \pm 5.6 \text { Control group: } \\
39.4 \pm 3.8\end{array}$ & 4.0 to 8.0 & 16 RRMS; 7 SPMS. \\
\hline Harris et al. (2016) & 6 & 4 & 2 & 36-64 Mean: 43 & 6.5 to 9.0 & 2 PPMS; 4 SPMS. \\
\hline Harris et al. (2018) & 20 & 14 & 6 & 27-65 Mean: 49 & 3.5 to 8.5 & 4 PPMS; 16 SPMS. \\
\hline Cohen et al. (2018) & 24 & 16 & 8 & Mean: 46.4 & 3.0 to 6.5 & 10 RRMS; 14 SPMS. \\
\hline Fernandez et al. (2018) & 30 & 21 & 9 & $31-61$ & 6.0 to 9.0 & 30 SPMS \\
\hline
\end{tabular}

using the Quality Assessment Tool for Before-After Studies with No Control Group (National Heart Lung and Blood Institute). For randomized-controlled trials, the methodological quality assessment was directly retrieved from the Critical Appraisal Skills Programmer (CASP) checklist. The checklist of BMJ Evidence-Based Medicine, except the fifth question, since did not fit our study, was used for the case report.

\section{Results}

\subsection{Design and samples}

In this study, 863 references were initially identified through database searching. In the first screening, 528 manuscripts were excluded (reviews, meta-analysis, protocols and other non-primary research articles). By further reading the titles and abstracts of the remaining 335 articles, 300 were excluded by the following criteria: other topics, animal studies or studies not written in English (Section 2.2). The full texts of the remaining 35 eligible articles were further analyzed. Finally, 10 studies were selected, due to their focus on the study of safety and/or efficacy of MSC therapies in MS. The flowchart of the review process is present in Fig. 1.

\subsection{Quality appraisal}

Tables 1-3 (see Appendix S1) present the results of the quality evaluation performed for the methodology of the uncontrolled clinical trials, randomized controlled clinical trials and a case report, respectively. Considering the results obtained and the number of red and green classifications, the quality of the methodology used in the uncontrolled clinical trial studies was considered good in three, fair in one and poor in the remaining three cases (Table 1). Both randomized clinical trials (Table 2) and the case report (Table 3) presented a good quality of the methodology used in their studies. However, one of the randomized clinical trials as a higher number of green classifications, and consequently, was considered of very good quality.

\subsection{Studies characteristics}

Ten studies were selected for this systematic review and their main characteristics are present in Table 4. Nine of the studies were clinical trials and one was a case report. In the clinical trials group, two are controlled and randomized. Moreover, one of those is triple blinded. The phases of clinical trials varied: five are phase I clinical trials, two are phase II clinical trials and two are phase I/II clinical trials. Despite all studies are based on the treatment of MS with MSCs, different tissue sources and routes of administration were used (Table 4). Seven of the studies used autologous bone marrow-derived (B)MSCs, one used autologous adipose-derived (A)MSCs, one used healthy human donor umbilical cord (UC)MSCs and the other used cells-derived from both autologous bone marrow and umbilical cord donors. Regarding their administration, three routes were used (Table 4). Three of the studies used an intravenous (IV) infusion, five administered the cells by the intrathecal route, one used both approaches and one used the intrathecal and intracisternal injections. The time period of follow up of the studies also varied (Table 4). The mean time period for all studies was about 24 months, being the maximum and minimum of follow up of 7.4 years and 6 months, respectively. Table S1 presents the inclusion and exclusion criteria of the selected studies.

\subsection{Population description}

161 participants were enrolled in the selected studies, being 52 male and 109 female. Their ages varied in the interval between 22 and 65 years. The clinical conditions of the participants were evaluated using the EDSS scale before study beginning. Different scores were obtained, being the minimum and maximum of 3.5 and 9, respectively. Although the MS clinical course was not described in one of the included studies (Karussis et al., 2010), the other studies involved 109 SPMS, 27 RRMS, and 10 PPMS patients. Table 5 presents the main characteristics of the patients enrolled in the selected studies.

\subsection{Main outcomes}

The outcomes obtained in the different studies were variable. In this systematic review, we focused on two, namely: the AEs of the therapy and the changes in the EDSS score (Table 6).

The analyzed studies reported some AEs (Table 6). The studies in which intrathecal injection was the selected administration route reported frequent headaches and low-grade fever. One case of encephalopathy with seizure and two cases of iatrogenic meningitis were also reported. For the studies that used IV infusion for cells administration, the most typical adverse effects reported were urinary tract infections and respiratory infections.

The analyses of the EDSS score (Table 6) were followed by their correlation with the clinical course of MS, the origin of MSCs and the sex of the patients. The randomized study of Fernandez et al. (2018) included 30 patients with SPMS, which were treated with AMSCs. The results did not show statistically significant differences in the mean EDSS score during the study. The randomized study of Li et al. (2014) was performed with RRMS and SPMS patients (70\% RRMS patients) and used UCMSCs for their treatment. The mean EDSS scores of the experimental group were statistically significantly lower than those of the control group (administration of anti-inflammatory drug and methylprednisolone). Both randomized studies had $70 \%$ of female participants.

In the uncontrolled clinical trials, five had participants with PPMS and SPMS. Four of those studies presented the changes in the EDSS score distributed for each clinical course. Among 42 patients with SPMS, 4 were not followed, 11 improved, 19 did not present any improvement and 8 worsened the EDSS score at the end of follow up. Analyzing the outcomes of the 6 PPMS patients, 2 improved, 1 maintained and 3 worsened their EDSS score. The clinical trial of Harris et al. 


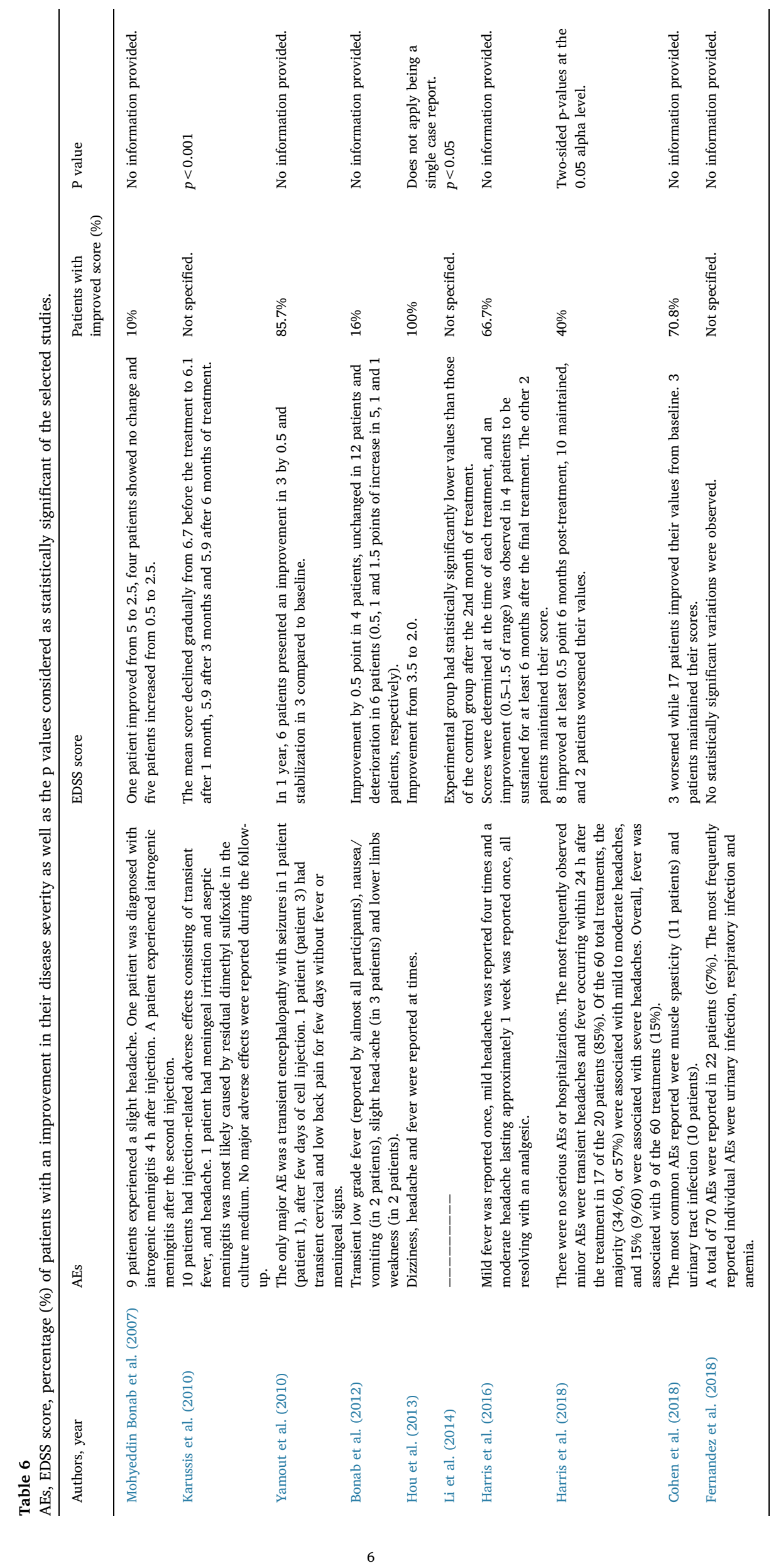


(2018) included 16 participants with SPMS and 4 with PPMS. Improvement in the EDSS score was obtained in 8, clinical stabilization in 10 and worsened scores were observed in 2 patients. The clinical trial of Cohen et al. (2018) was performed with 10 RRMS patients and 14 SPMS patients. 7 of the participants presented an improvement in the EDSS score, 4 maintained their values and 3 worsened their clinical condition. Finally, in Karussis et al. (2010) this information was not available. The percentage of females varied from $53 \%$ to $76 \%$ in the uncontrolled clinical trials. In three of these studies, the EDSS score was described for every single patient and related to sex. Therefore, the data obtained for 30 females and 11 males was analyzed. Regarding females patients, 6 improved, 14 did not show differences and 10 worsened their EDSS score. Concerning male patients, 3 improved, 4 maintained and 1 worsened their EDSS score ( 3 were not followed). The other uncontrolled clinical trial did not present sex stratification of the results. Considering all the results, it can be concluded that in the uncontrolled clinical trials: 48 patients improved, 39 maintained and 16 worsened their EDSS score throughout the study. It is also important to highlight that all these studies used BMSCs therapies.

In the case report, a male patient with RRMS treated with both UCMSCs and BMSCs presented a decrease in his EDSS score.

\section{Discussion}

This systematic review results of the growing interest and importance of stem cell therapies for a seriously debilitating condition that affects millions of patients worldwide, namely MS. Notwithstanding different types of stem cells can be used, MSCs have been widely investigated for their multi-lineage potential, immunomodulatory effect and limited immunogenicity, for instance (Mohyeddin Bonab et al., 2007). Therefore, this systematic review investigated the safety, tolerability, and efficacy of MSCs therapies in MS.

The selected manuscripts have, overall, a good quality of the methodology used in their studies. However, some limitations were identified in most studies, such as their unblinded and uncontrolled design, the small sample size and the short time period time of follow up. Furthermore, one of the manuscripts is a case report and, consequently, needs to be cautiously analyzed.

The results presented in this systematic review reinforced the safety profile of MSCs therapies. Despite two meningitis and one transient encephalopathy were described in the studies using intrathecal injection, the authors did not relate it with the treatment, but with the administration route. Minor AEs reported in intrathecal injection and in IV infusion can be considered irrelevant if the treatment proves its efficacy. However, a conclusion about safety in these studies is limited, due to the small mean time period of follow up and sample size, raising questions such as long term or rare AEs.

MSCs are considered non-immunogenic, however, some studies indicated that repeated administration stimulates rejection. Comparing the autologous stem cells with allogeneic from human healthy donors, the use of autologous avoids potential concerns with immunogenic reactions or disease transmission (Cohen et al., 2018). The manuscripts reviewed with human healthy donors MSCs do not report any rejection even though multiple administrations were performed. This issue needs to be further investigated given the small number of studies selected using donor cells.

Despite the majority of the manuscripts were designed to establish the safety and tolerability of MSC therapies, they also analyze their efficacy as an outcome. Therefore, definitive conclusions cannot be obtained for MSC therapies efficacy, but the studies reviewed show the potential therapeutic benefits of these treatments for MS.

As previously mentioned, MS presents different clinical courses with different responses to therapies. Nowadays, RRMS has several drugs to target the inflammatory component of the disease with good clinical outcomes. However, PPMS and SPMS, the more severe forms of the disease, have very limited therapeutic options (Fernandez et al., 2018;
Harris et al., 2016). This can justify the high enrolment of PPMS and SPMS patients in the selected studies, despite affecting a lower number of MS patients. As RRMS usually responds better to the treatment, it would be expected to obtain better results in the RRMS patients compared to PPMS and SPMS patients. The population analyzed in this review does not have sufficient patients with PPMS or RRMS to compare the efficacy of the therapy in these different clinical courses. Clinical trials that aim at establishing which clinical course has a better response to MSCs treatment are needed to obtain more robust and definitive conclusions.

The optimal route of MSCs administration in MS patients remains debatable. Some studies claimed that IV infusion may be equally effective as other administration routes. Other studies declared that after an IV infusion the number of cells available to migrate to the CNS is reduced, since they can be trapped in the lungs, for example. Therefore, intrathecal administration may be more effective (Cohen et al., 2018; Karussis et al., 2010). After reviewing the selected manuscripts, it is possible to conclude that intrathecal injection of MSCs presented therapeutic efficacy. However, a comparison between the efficacy of IV and intrathecal infusions cannot be drawn, since the studies administered different types of MSCs (e.g. AMSCs and UCMSCs) and there is a lack of studies using both intrathecal and IV injections. Moreover, one of the selected studies that use IV infusion is a case report. To overcome these limitations and compare these two administration routes more effectively, blinded, controlled and randomized clinical trials with an intrathecal injection group and an IV infusion group should be conducted.

In this systematic review, as previously stated, we analyzed studies that used different MSCs types. Despite autologous AMSCs can be obtained by a less invasive procedure than bone marrow aspirations (Fernandez et al., 2018), only one study used the adipose tissue to obtain the cells to treat MS patients. Consequently, the absence of robust data unable draws a clear statement about the efficacy of AMSCs vs BMSCs. This difficulty also arises for UCMSCs. However, the case report reviewed claims that human UCMSCs may be a safe, effective and more practical source of stem cells for the MS treatment (Hou et al., 2013). Autologous BMSCs were the type of stem cells more prevalent in the manuscripts considered. These cells can be readily and safely obtained from adult bone marrow, even from patients with advanced disease stages (Karussis et al., 2010). Nevertheless, the different types of MSCs were not yet systematically investigated for its potency in the MS treatment. The cohorts of patients reported in the selected manuscripts consisted of people with moderate to the high levels of disability (EDSS scores of 3.5-9). Therefore, the generalization of these outcomes to patients with less severe disease stages is not possible. The diversity in methods applied in the different studies further complicates drawing definite conclusions. Those issues should be considered since both individually and collectively data influence the obtained conclusions.

\section{Conclusions}

This systematic review reinforces that MSCs transplantation is a safe and tolerable therapy for MS patients. Moreover, the potential benefits of MSC therapies were further consolidated on the selected manuscripts, however, stronger evidence in the literature is needed to conclude about their really efficacy and potency. Larger randomized, placebo controlled and double blinded clinical trials are required to translate these treatments into the clinical practice to benefit millions of patients suffering from this highly debilitating condition.

\section{Funding}

This work was not supported by funding agencies.

Appendix S1. Questions of Quality Assessment Tool for BeforeAfter Studies with No Control Group, CASP checklist and checklist of BMJ Evidence-Based Medicine for Tables 1, 2 and 3, respectively. 
Table S1. Inclusion and exclusion criteria of the selected studies.

\section{Declaration of Competing Interests}

The authors declare no conflicts of interest pertinent to this study.

\section{Supplementary materials}

Supplementary material associated with this article can be found, in the online version, at doi:10.1016/j.msard.2019.101860.

\section{References}

Iwanowski, P., Losy, J., 2015. Immunological differences between classical phenothypes of multiple sclerosis. J. Neurol. Sci. 349:, 10-14.

Keegan, B.M., Noseworthy, J.H., 2002. Multiple sclerosis. Annu. Rev. Med. 53:, 285-302.

Thompson, A.J., Baranzini, S.E., Geurts, J., Hemmer, B., Ciccarelli, O, 2018. Multiple sclerosis. Lancet 391:, 1622-1636.

Giovannoni, G., Butzkueven, H., Dhib-Jalbut, S., et al., 2016. Brain health: time matters in multiple sclerosis. Mult. Scler. Relat. Disord 9, S5-s48 Suppl 1.

Scalfari, A., Neuhaus, A., Daumer, M., Muraro, P.A., Ebers, G.C, 2014. Onset of secondary progressive phase and long-term evolution of multiple sclerosis. J. Neurol. Neurosurg. Psych. 85:, 67-75.

Ontaneda, D., Thompson, A.J., Fox, R.J., Cohen, J.A, 2017. Progressive multiple sclerosis: prospects for disease therapy, repair, and restoration of function. Lancet 389: 1357-1366.

Ramien, C., Taenzer, A., Lupu, A., et al., 2016. Sex effects on inflammatory and neurodegenerative processes in multiple sclerosis. Neurosci. Biobehav. Ver. 67, 137-146.

Harbo, H.F., Gold, R., Tintoré, M, 2013. Sex and gender issues in multiple sclerosis. Ther. Adv. Neurol. Disord. 6:, 237-248.

Cohen, J.A., Imrey, P.B., Planchon, S.M., et al., 2018. Pilot trial of intravenous autologous culture-expanded mesenchymal stem cell transplantation in multiple sclerosis. Mult. Scler. 24: 501-511.

Li, J.F., Zhang, D.J., Geng, T., et al., 2014. The potential of human umbilical cord-derived mesenchymal stem cells as a novel cellular therapy for multiple sclerosis. Cell Transplant 23, S113-S122 Suppl 1.

Comi, G., Radaelli, M., Soelberg Sorensen, P, 2017. Evolving concepts in the treatment of relapsing multiple sclerosis. Lancet 389:, 1347-1356.

Chandran, S., Compston, A., 2005. Neural stem cells as a potential source of oligodendrocytes for myelin repair. J. Neurol. Sci. 233:, 179-181.

Prineas, J.W., Connell, F., 1979. Remyelination in multiple sclerosis. Ann. Neurol. 5:, 22-31.

Juric, M.K., Ghimire, S., Ogonek, J., et al., 2016. Milestones of hematopoietic stem cell transplantation - From First human studies to current developments. Front. Immunol. $7:, 470$.

McLauchlan, D., Robertson, N.P., 2018. Stem cells in the treatment of central nervous system disease. J. Neurol. 265:, 984-986.

Shroff, G., 2018. A review on stem cell therapy for multiple sclerosis: special focus on human embryonic stem cells. Stem. Cells Cloning 11:, 1-11.

Cuascut, F.X., Hutton, G.J., 2019. Stem cell-based therapies for multiple sclerosis: current perspectives. Biomedicines 7.

Aharonowiz, M., Einstein, O., Fainstein, N., Lassmann, H., Reubinoff, B., Ben-Hur, T, 2008. Neuroprotective effect of transplanted human embryonic stem cell-derived neural precursors in an animal model of multiple sclerosis. PLoS One 3:, e3145.

Shroff, G., 2015. Human embryonic stem cell for the treatment of multiple sclerosis: a case report. Case Rep. Int.. 4:, 38-42.

Muraro, P.A., Martin, R., Mancardi, G.L., Nicholas, R., Sormani, M.P., Saccardi, R, 2017. Autologous haematopoietic stem cell transplantation for treatment of multiple sclerosis. Nat. Rev. Neurol. 13:, 391.

Laterza, C., Merlini, A., De Feo, D., et al., 2013. iPSC-derived neural precursors exert a neuroprotective role in immune-mediated demyelination via the secretion of LIF. Nat. Commun. 4: 2597.

Thiruvalluvan, A., Czepiel, M., Kap, Y.A., et al., 2016. Survival and functionality of human induced pluripotent stem cell-derived oligodendrocytes in a nonhuman primate model for multiple sclerosis. Stem. Cells Transl. Med. 5:, 1550-1561.

Okano, H., Nakamura, M., Yoshida, K., et al., 2013. Steps toward safe cell therapy using induced pluripotent stem cells. Circ Res. 112:, 523-533.

Lukomska, B., Stanaszek, L., Zuba-Surma, E., Legosz, P., Sarzynska, S., Drela, K, 2019. Challenges and controversies in human mesenchymal stem cell therapy. Stem Cells Int. 2019, 10.

Colpo, G.D., Ascoli, B.M., Wollenhaupt-Aguiar, B., et al., 2015. Mesenchymal stem cells for the treatment of neurodegenerative and psychiatric disorders. An Acad Bras Cienc 87:, 1435-1449.

Uccelli, A., Laroni, A., Freedman, M.S, 2011a. Mesenchymal stem cells for the treatment of multiple sclerosis and other neurological diseases. Lancet Neurol 10:, 649-656.

Zheng, Y., Huang, C., Liu, F., Lin, H., Yang, X., Zhang, Z, 2017. Comparison of the neuronal differentiation abilities of bone marrow-derived and adipose tissue-derived mesenchymal stem cells. Mol. Med. Rep. 16:, 3877-3886.

Jorgensen, C., Noel, D., 2011. Mesenchymal stem cells in osteoarticular diseases. Regen. Med. 6:, 44-51.

Jiang, H., Zhang, Y., Tian, K., Wang, B., Han, S, 2017. Amelioration of experimental autoimmune encephalomyelitis through transplantation of placental derived mesenchymal stem cells. Sci. Rep. 7 41837-41837.

Bai, L., Lennon, D.P., Eaton, V., et al., 2009. Human bone marrow-derived mesenchymal stem cells induce Th2-polarized immune response and promote endogenous repair in animal models of multiple sclerosis. Glia 57:, 1192-1203.

Yan, L., Jiang, B., Niu, Y., et al., 2018. Intrathecal delivery of human ESC-derived mesenchymal stem cell spheres promotes recovery of a primate multiple sclerosis model. Cell Death Dis. 4:, 89.

Kassis, I., Grigoriadis, N., Gowda-Kurkalli, B., et al., 2008. Neuroprotection and immunomodulation with mesenchymal stem cells in chronic experimental autoimmune encephalomyelitis. JAMA Neurol. 65:, 753-761.

Keirstead, H.S., 2005. Stem cells for the treatment of myelin loss. Trends Neurosci. 28: 677-683.

Ardeshiry Lajimi, A., Hagh, M.F., Saki, N., Mortaz, E., Soleimani, M., Rahim, F, 2013. Feasibility of cell therapy in multiple sclerosis: a systematic review of 83 studies. Int. J. Hematol. Oncol. Stem Cell Res. 7:, 15-33.

Uccelli, A., Laroni, A., Freedman, M.S, 2011b. Mesenchymal stem cells for the treatment of multiple sclerosis and other neurological diseases. Lancet Neurol 10:, 649-656.

Fernandez, O., Izquierdo, G., Fernandez, V., et al., 2018. Adipose-derived mesenchymal stem cells (AdMSC) for the treatment of secondary-progressive multiple sclerosis: a triple blinded, placebo controlled, randomized phase i/ii safety and feasibility study. PLoS One 13:, e0195891.

Hou, Z.L., Liu, Y., Mao, X.H., et al., 2013. Transplantation of umbilical cord and bone marrow-derived mesenchymal stem cells in a patient with relapsing-remitting multiple sclerosis. Cell Adh. Migr. 7:, 404-407.

Mohyeddin Bonab, M., Yazdanbakhsh, S., Lotfi, J., et al., 2007. Does mesenchymal stem cell therapy help multiple sclerosis patients? report of a pilot study. Iran J. Immunol 4:, 50-57.

Karussis, D., Karageorgiou, C., Vaknin-Dembinsky, A., et al., 2010. Safety and immunological effects of mesenchymal stem cell transplantation in patients with multiple sclerosis and amyotrophic lateral sclerosis. Arch Neurol. 67:, 1187-1194.

Yamout, B., Hourani, R., Salti, H., et al., 2010. Bone marrow mesenchymal stem cell transplantation in patients with multiple sclerosis: a pilot study. J. Neuroimmunol 227:, 185-189.

Bonab, M.M., Sahraian, M.A., Aghsaie, A., et al., 2012. Autologous mesenchymal stem cell therapy in progressive multiple sclerosis: an open label study. Curr. Stem. Cell Res. Ther 7:, 407-414.

Harris, V.K., Vyshkina, T., Sadiq, S.A, 2016. Clinical safety of intrathecal administration of mesenchymal stromal cell-derived neural progenitors in multiple sclerosis. Cytotherapy 18:, 1476-1482.

Harris, V.K., Stark, J., Vyshkina, T., et al., 2018. Phase i trial of intrathecal mesenchymal stem cell-derived neural progenitors in progressive multiple sclerosis. E Bio. Med. 29:, 23-30. 